\title{
BIODIVERSIDAD VEGETAL Y CIUDAD: APROXIMACIONES DESDE LA ECOLOGÍA URBANA ${ }^{1}$
}

\author{
Josep Padullés Cubino \\ Departamento de Geografia/Institut de Medi Ambient. Universidad de Girona \\ josep.padulles@udg.edu \\ Josep Vila Subirós \\ Departamento de Geografía/ Institut de Medi Ambient. Universidad de Girona \\ josep.vila@udg.edu \\ Carles Barriocanal Lozano \\ Departamento de Geografía y Análisis Geográfico Regional. Universidad de Barcelona carles.barriocanal@ub.edu
}

\section{RESUMEN}

La ecología urbana analiza los procesos y las interacciones de los ecosistemas urbanos con su entorno biofísico y humano más inmediato. Estos ecosistemas albergan altos niveles de biodiversidad a la vez que proporcionan bienes y servicios a la sociedad. El propósito de este estudio es elaborar un marco teórico a partir de la revisión bibliográfica de algunos de los trabajos más relevantes sobre ecología y vegetación urbana. De este modo se articula un escenario que permite acercarse a las áreas verdes urbanas, y en concreto a los jardines domésticos, desde una perspectiva más integradora.

Palabras clave: ecología urbana, biodiversidad vegetal, ciudad, jardines domésticos.

\footnotetext{
ABSTRACT

Urban ecology examines processes and interactions between urban ecosystems and their biophysical and immediate human environment. These ecosystems host vegetated areas with high levels of biodiversity and also provide several goods and services to society. The purpose of this study is to develop a theoretical framework based on a review of relevant

Fecha de recepción: marzo 2013.

Fecha de aceptación: diciembre 2013.

1 Nombre del proyecto: «Nuevas pautas de consumo y gestión del agua en espacios urbanoturísticos de baja densidad. el caso de la Costa Brava (Gerona)». Ref: CSO2010-17488. Entidad financiadora: Ministerio de Ciencia e Innovación. Investigador principal: Anna Ribas Palom. Josep Padullés Cubino dispone de una beca tipo FPI para realizar su PhD. Ref: BES-2011-046475.
} 
literature on urban ecology and urban vegetation. A scenario that allows an integrative approach to urban ecosystems and especially to domestic gardens is developed.

Keywords: urban ecology, plant biodiversity, city, domestic gardens.

\section{INTRODUCCIÓN}

El proceso de crecimiento urbano a nivel mundial se ha visto multiplicado en los últimos 60 años hasta el punto que más del 50 por ciento de población de todo el mundo vive hoy en ciudades (United Nations, 2013). La tasa de crecimiento es especialmente acelerada en los países en vías de desarrollo, y la tendencia global puede tener consecuencias drásticas sobre la preservación del entorno natural y sus procesos (Wu et al., 2003). Para hacer frente a estos retos, los nuevos estudios de ecología deben incluir el elemento humano como un factor de cambio importante en los ecosistemas urbanos (Hope et al., 2003).

En este contexto, la ecología urbana es la ciencia que estudia los ecosistemas urbanos y que da un paso al frente respecto la ecología tradicional para incorporar la variable humana como primer agente de evolución y cambio (Alberti et al., 2003). En los últimos años, esta ciencia ha evolucionado de tal manera que ha permitido integrar diferentes disciplinas para un mejor entendimiento de los procesos y factores que condicionan los ecosistemas urbanos. Además, se ha dado un aumento considerable de este tipo de estudios (Goddard et al., 2009; Pickett et al., 2011) con influencia sobre el planeamiento urbano sostenible y el desarrollo de infraestructuras urbanas ecoeficientes (Hooves y Robinson, 2005).

Un campo de especial interés para la ecología urbana es el estudio de las áreas verdes de las ciudades y su relación con el entorno socioeconómico más inmediato (Chivian y Bernstein, 2008). Estos espacios juegan un papel fundamental y cumplen diferentes funciones; desde proporcionar beneficios intangibles tales como el bienestar social, hasta la obtención de alimentos y servicios ambientales como la renovación del aire, la reducción de ruido o la reducción de la temperatura urbana global (Costanza et al., 1997).

Un tipo concreto de áreas verdes son los denominados jardines domésticos, los cuales pueden llegar a ocupar aproximadamente un tercio de la superficie de las áreas urbanas en zonas residenciales dispersas (Gaston et al., 2005; Loram et al., 2007; Mathieu et al., 2007), también en el contexto mediterráneo (García, 2012; Domene y Saurí, 2003). Este hecho evidencia la relevancia que tienen este tipo de ecosistemas en los usos y cubiertas de suelo urbano. Asimismo, cobra importancia la necesidad de analizar los mecanismos que configuran estos espacios, a menudo no tan ligados a factores naturales sino a una combinación de diferentes factores demogràficos y socioeconómicos.

Los motivos que enfatizan la importancia de incluir los jardines privados en los estudios de ecología urbana son diversos. Así, por ejemplo, estos espacios pueden actuar como hábitat conector para cierto número de especies en contraste con otros ecosistemas urbanos aislados por procesos de fragmentación y que no realizan esta función (Colding, 2007). Por otra parte, los jardines, y en conjunto la horticultura, actúan también como punto de entrada de especies exóticas que pueden tener capacidad para naturalizarse en nuevos hábitats (Reichard y White, 2001) provocando incluso la extinción de especies a nivel local (Smith et al., 2006). 
Los objetivos del presente trabajo son (1) establecer un marco teórico para la aplicación de los métodos de la ecología urbana en el estudio de la flora urbana, (2) analizar los patrones que determinan la composición vegetal en áreas verdes urbanas y describir la importancia ecológica y social que desempeñan, y (3) finalmente reivindicar el papel de los jardines domésticos como un ecosistema de interés para la ecología urbana y la sensibilización ambiental.

\section{METODOLOGÍA}

La primera parte del trabajo se fundamenta en la recopilación de publicaciones relevantes en el ámbito de la ecología y la biodiversidad vegetal urbana. Para ello se realizaron búsquedas en los títulos, resúmenes y palabras clave en distintas bases de datos como Web of Science o Scopus y otras revistas significativas no incluidas en estas bases de datos (por ejemplo, Urban Ecosystems). Junto con esta búsqueda se identificaron otras publicaciones de referencia dentro de los artículos obtenidos por el primer método. Además, se llevó también a cabo una búsqueda exhaustiva en bibliotecas para documentos de interés en soporte analógico.

En la segunda parte del trabajo, y a partir de toda la documentación obtenida, se empleó un análisis integrado de todas las publicaciones para dar respuesta a los objetivos planteados. Para sistematizar la información se optó por dividir el trabajo en cuatro secciones principales. En la primera de ellas se recoge un análisis de bibliografía vinculada a la ecología urbana, haciendo especial hincapié en sus conceptos básicos, términos clave y las metodologías más relevantes para el estudio de la vegetación urbana. La segunda sección trata un ámbito concreto de estudio para la ecología urbana como es la biodiversidad vegetal en áreas verdes urbanas para evaluar las causas que determinan su evolución y su importancia a nivel ecológico. A continuación, y en la tercera sección, se realiza un salto de escala para analizar el caso particular de los jardines domésticos como parte de las áreas verdes con el fin de reivindicar su rol en la conservación biológica y sensibilización ambiental. Finalmente, y ya en el apartado de conclusiones, se integran y se enlazan las ideas presentadas en las secciones anteriores y se construye un marco de trabajo para abordar el estudio de la biodiversidad vegetal en áreas urbanas desde la perspectiva de la ecología urbana.

\section{IMPORTANCIA Y SIGNIFICADO DE LA ECOLOGÍA URBANA}

\section{III.1. Evolución de la Ecología Urbana y aproximaciones al conocimiento de sus objetivos y metodología}

El progresivo aumento de la población urbana a nivel mundial tiene consecuencias directas sobre el consumo de suelo por parte de edificios o infraestructuras que a menudo se traduce en una pérdida de hábitats naturales (Kendle y Forbes, 1997), de biodiversidad (Vitousek et al., 1997), o en la contaminación del medio ambiente (Rueda, 1995). Para hacer frente a estos retos, la ecología urbana puede jugar también un papel fundamental.

Durante muchos años, la ecología ha centrado su interés en las dinámicas y procesos del medio natural de espaldas a la realidad urbana y a menudo sin integrar la componente humana 
como agente modelador de estos procesos (McDonnell y Pickett, 1990). Desde principios del siglo XX, sin embargo, la idea de incluir el factor humano como un elemento más de los ecosistemas urbanos ha crecido en importancia (Adams, 1935; Tansley, 1935; Margalef, 1974). En pleno siglo XXI estos conceptos se encuentran plenamente integrados (McIntyre et al., 2000; Luck y Wu, 2002), gracias, entre otros, al uso de nuevas tecnologías como la teledetección que permite la obtención de datos ambientales a gran escala (Mathieu et al., 2007).

En la nueva ecología urbana la «urbanización» se convierte en un proceso social al mismo tiempo que ecológico (Parlange, 1998). Así, la integración de las dos vertientes de la ciencia contemporánea, la natural y la social, es crucial en el análisis de los ecosistemas urbanos, haciendo de la ecología urbana una disciplina realmente interdisciplinaria (Walbridge, 1997).

Wu (2008) distingue cinco perspectivas ecológicas según la aproximación de los diferentes estudios de ecología urbana: la ecología en las ciudades (EIC) (1), la ecología de las ciudades entendidas como estructuras socioeconómicas (EOC-S; 2 ), y tres perspectivas que se desprenden de la ecología de las ciudades entendidas como ecosistemas (EOC-E; 3), y que el autor llama perspectiva de los sistemas urbanos (3), perspectiva integrada de los ecosistemas urbanos (4) y perspectiva de la ecología del paisaje urbano (5) (Figura 1).

Figura 1

ESQUEMA DE LAS CINCO PERSPECTIVAS ECOLÓGICAS EN LOS ESTUDIOS DE ECOLOGÍA URBANA

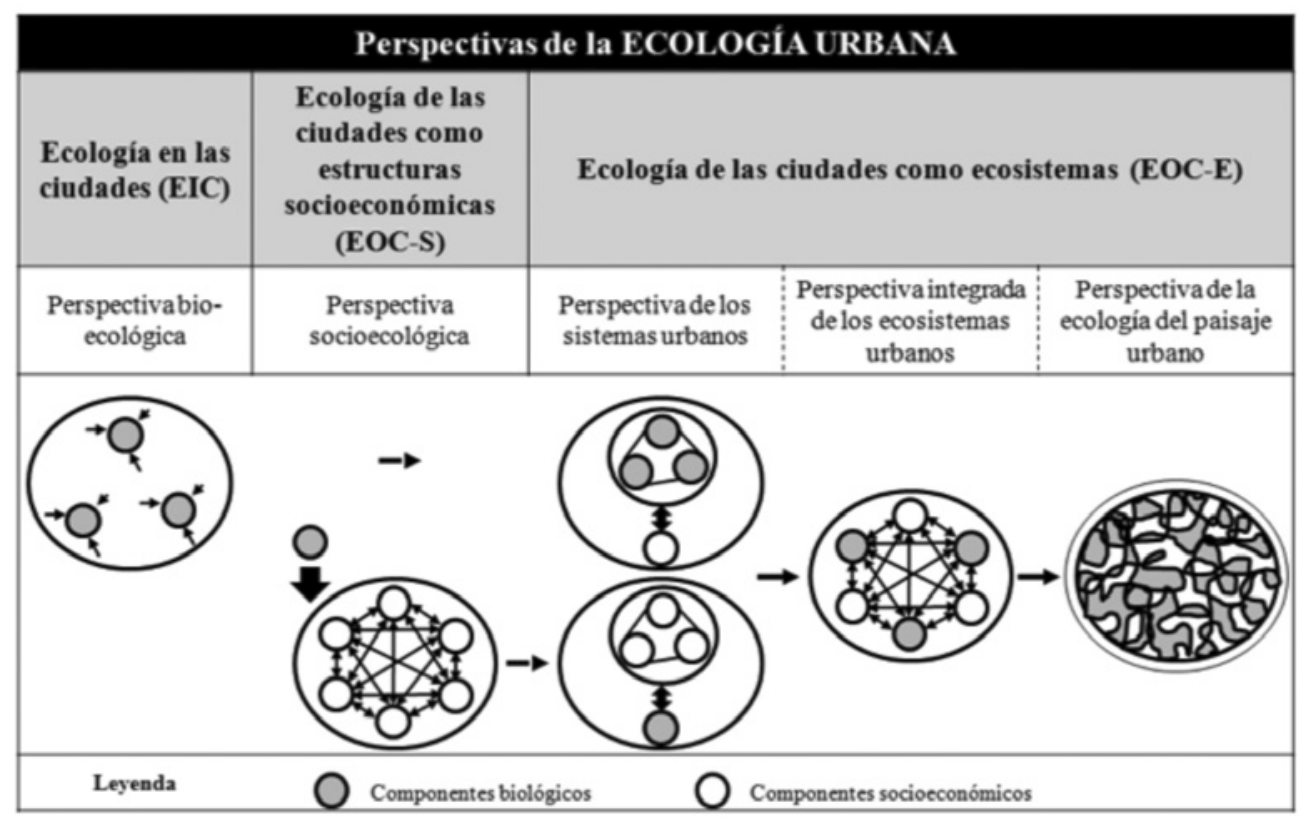

Fuente: Elaborado a partir de Wu, 2008.

En la primera de las aproximaciones, la ciudad no es vista como un ecosistema en sí misma, sino que el interés ecológico se centra en el conocimiento de la naturaleza dentro de las áreas urbanas poniendo especial énfasis en hábitats y grupos de organismos específicos. 
Este es un ámbito de investigación muy vinculado a la biogeografía urbana. La segunda de las perspectivas, por su parte, incorpora los principios ecológicos en un sistema urbano considerado eminentemente socioeconómico. La tercera perspectiva, llamada de los sistemas urbanos, ve la vertiente ecológica y socioeconómica como dos subconjuntos que mantienen relaciones pero que no se encuentran integrados entre sí. Esta integración tiene lugar en la cuarta de las perspectivas. En la última de las aproximaciones, la perspectiva de la ecología del paisaje urbano, todas las demás perspectivas se integran y se complementan. Además, también en este punto cobra fuerza la necesidad de trabajar a diferentes escalas para analizar la heterogeneidad de las parcelas de los ecosistemas urbanos.

Las aproximaciones interdisciplinarias y aquellas que reúnen no sólo el sector académico, sino también los sectores no académicos, pueden ayudar a crear una plataforma desde donde los problemas ambientales, y sociales, converjan en la búsqueda de una solución conjunta (Cilliers, 2010). Así, por ejemplo, la incorporación de las técnicas de la ecología del paisaje permite tomar en consideración elementos como la escala de trabajo, la relación entre matriz y parcela, o el diseño de conectores ecológicos, todas ellas de gran interés para la ecología urbana (Wu, 2008).

A nivel internacional diferentes estudios han incorporado ya la vertiente más social en los métodos clásicos de la ecología. Así, para explicar los patrones de configuración de la biodiversidad en ecosistemas urbanos, algunos optan por incluir factores como la preferencia humana (Acar et al., 2007; Kendal et al., 2012), la relevancia de los factores socioeconómicos (Marco et al., 2010; Bigirimana et al., 2012), o las dinámicas demográficas (Roy Chowdhury et al., 2011).

En España distintos autores han resaltado el papel de la ecología urbana y la necesidad de incorporarla eficientemente en el planeamiento urbano. Cabe destacar los trabajos de Rueda (1995) y Terradas (2001), los cuales aparte de ofrecer reflexiones y perspectivas generales en torno a esta disciplina y sus métodos, utilizan referentes claros para detallar los procesos en el metabolismo de las ciudades. Sin duda las grandes aglomeraciones urbanas, como Barcelona y su área metropolitana, han sido objeto principal de análisis y estudio (Rueda 1995; Barracó et al., 1999). No obstante, en general se han realizado aproximaciones particulares para un número muy limitado de ciudades y para temáticas muy concretas. Feria y Santiago (2009) analizan de forma conceptual y metodológica los servicios ecológicos aplicados al espacio libre en áreas urbanas. De este modo, sitúan el foco de atención en las funciones que desarrollan los procesos naturales para mejorar y hacer más sostenible el medio ambiente urbano. Para ilustrar la relevancia de incluir estas funciones en el planeamiento urbano sostenible, los autores describen algunas experiencias recientes de ordenación en el contexto español como los planes de las áreas metropolitanas de Sevilla o Córdoba. Otras aproximaciones han ido vinculadas a la ecología del paisaje con trabajos sobre conectividad ecológica y el papel que juegan las grandes áreas metropolitanas en este proceso (Pino y Marull, 2012; Rodríguez-Rodríguez, 2012). También la biodiversidad y los efectos que los factores humanos tienen sobre las comunidades vegetales y animales han centrado distintos objetivos de investigación (ej. Buján et al., 1998; Dana et al., 2002; Murgui, 2009; Mendes et al., 2011). Sin embargo, gran número de hábitats y comunidades de los espacios urbanos siguen siendo aún desconocidos, y la ecología urbana se presenta como una disciplina con un amplio campo de investigación y desarrollo para hacer frente a estos retos. 


\section{III.2. Métodos de la ecología urbana para el análisis de la estructura vegetal: definición de tér- minos, clasificaciones y patrones}

El primer paso para determinar el ámbito de aplicación de la ecología urbana pasa por establecer una distinción clara entre aquellos espacios considerados «urbanos» de aquellos excluidos de dicha categoría. El uso del término «urbano», en el contexto de la ecología urbana, y en general de las ciencias naturales, está sujeto a diversas interpretaciones y definiciones, lo que complica la comparación de resultados entre estudios de temáticas muy similare. Ante estos inconvenientes, McIntyre et al. (2000) sugieren que cada uno de los estudios de ecología urbana incorpore su propia definición del concepto urbano y lo describa según sus características socioeconómicas, culturales, demográficas o geográficas para facilitar las comparaciones y la reproducibilidad de los estudios. Algunas publicaciones recientes han demostrado el potencial en el uso de diferentes gradientes urbanos para medir y afinar en el concepto de lo «urbano» (ej. Dow, 2000; Luck y Wu, 2002; Marco et al., 2008). Ahora bien, esta nueva metodología supone otro problema y es que la cuantificación de una variable apropiada para un estudio puede no serlo para otro, ya sea porque los intereses de la investigación son distintos o bien porque también lo es la escala de trabajo.

Una vez establecida la separación justificada entre las áreas consideras «urbanas» de las «no urbanas», el estudio de los ecosistemas urbanos centra su interés en la intersección entre los procesos biofísicos y sociales. Para lograr este fin, los marcos de trabajo y las metodologías utilizadas por la ecología urbana deben nutrirse de las técnicas de diferentes disciplinas con el fin de afinar en sus conclusiones. Dow (2000) afirma que las ciencias sociales a menudo conciben los asentamientos urbanos según las funciones que éstos desempeñan en el territorio (económica, política y cultural) e interpretan el medio físico como un paisaje visual simbólico o como un producto de los procesos de planeamiento. Por el contrario, los ecólogos tradicionalmente han destinado sus esfuerzos hacia áreas no urbanas, o aquellas con bajo impacto antrópico, mientras que los ecosistemas urbanos han quedado descuidados. Para tratar estos asuntos, la ecología urbana ha apostado por ciertos métodos descritos a continuación, haciendo especial énfasis en el uso de clasificación de matrizes y de patrones en forma de gradientes.

Una de las técnicas empleadas por la ecología urbana, y que deriva de las técnicas tradicionales de la ecología del paisaje, es el uso de la clasificación de la matriz urbana. Mediante este procedimiento, y aplicable a diferentes escalas, las ciudades son descompuestas en un gran mosaico de fragmentos urbanos, fragmentos vegetados y otros usos del suelo (Cadenasso et al., 2007). Posteriormente, con el uso de diferentes índices y herramientas de medición, se pueden cuantificar determinados procesos y dinámicas territoriales. Estos sistemas de clasificación se han visto beneficiados recientemente por los avances en las tecnologías de la teledetección como las imágenes satélite multiespectrales que permiten análisis de muy alta resolución. Un ejemplo es el trabajo de Mathieu et al. (2007), el cual clasifica de forma automática, y para la ciudad de Dunedin (Nueva Zelanda), más del 90\% de los jardines urbanos. Las técnicas en teledetección, además, también comienzan a tener aplicación en la predicción de la riqueza de especies de fauna en ambientes urbanos. Así, algunos estudios han llegado a prever la riqueza y distribución de ciertas especies de aves a partir de índices de vegetación, como el NDVI (Normalized Difference Vegetation Index), obtenidos a partir 
de sensores remotos y que permiten evaluar si el objetivo que se observa contiene vegetación viva (ej. Johnson et al. 1998; Bino et al., 2008). Para el análisis de la biodiversidad urbana en ciudades, Boada y Sànchez $(2011 ; 2012)$ proponen la clasificación de la estructura urbana en tres categorías: mundo gris, mundo verde y mundo azul. Estas, a su vez, deben clasificarse en una serie de biotopos.

El uso de patrones en el campo de la ecología urbana es también una herramienta ampliamente utilizada para cuantificar la relación entre los procesos ecológicos y la estructura de las ciudades. Los efectos de los patrones de desarrollo urbano sobre las funciones de los ecosistemas ha sido bien documentada por Alberti (2005). Ahora bien, un caso especial de patrones avalados por gran número de trabajos científicos son los gradientes. Según McDonnell y Pickett (1990) un gradiente sucede cuando existe una variación, o un cambio ambiental, que varía de forma ordenada y regular en el tiempo o en el espacio. Uno de los ejemplos más citados sobre la eficiencia de los gradientes es el estudio de la vegetación según la altitud (Whittaker, 1967).

La aplicación de los gradientes en el ámbito urbano puede ayudar a entender mejor las interacciones entre el desarrollo urbano y la estructura de los sistemas ecológicos y sociales (Alberti et al., 2001). Así, por ejemplo, este método permite estudiar las respuestas y los cambios de las comunidades vegetales frente a cambios ambientales graduales (Du Toit y Cilliers, 2011), o relacionar una determinada organización espacial según los procesos urbanos y ecológicos que se han llevado a cabo (Luck y Wu, 2002). McDonnell y Hahs (2008) han analizado más de 200 trabajos que utilizan gradientes para tratar el impacto de la urbanización sobre los organismos. Uno de los gradientes más utilizados satisfactoriamente es el gradiente urbano-rural, el cual ordena los espacios según su densidad urbana para explicar variaciones en las comunidades vegetales o animales, entre otros (Luck y Wu, 2002; McDonnell y Hahs, 2008; Du Toit y Cilliers, 2011).

Los tipos de gradientes más comunes son los llamados gradientes complejos, y se definen por contar con más de una variable de contraste. En estos casos, es necesario cuantificar, por separado, cada una de las variables para reconstruir un gradiente indirecto que pueda explicar el máximo de variabilidad posible (McIntyre et al., 2002). Este elemento es de especial importancia en las ciudades ya que los ambientes urbanos son muy heterogéneos y a menudo la cuantificación de los gradientes es dificultosa y está sujeta a diversos parámetros. Utilizando este recurso es posible reducir la subjetividad dentro de los resultados (Hahs y McDonnell, 2006) y establecer comparaciones de gradientes entre ciudades de todo el mundo que utilicen la misma metodología. Un caso particular de gradientes, y que merecen un trato diferencial, son aquellos que parten de datos socioeconómicos como base para su confección. La inclusión de elementos sociales cuantificables en la elaboración de los gradientes puede fortalecer los resultados y ayudar a predecir patrones de biodiversidad urbana (Collins et al., 2000; Kinzig et al., 2005).

Diversos estudios han demostrado que la influencia de los factores socioeconómicos tiene consecuencias directas sobre la diversidad florística de los espacios urbanos (ej. Hope et al., 2003; Kinzig et al., 2005). Los recursos de que dispone un grupo poblacional pueden limitar o mejorar las condiciones ambientales para la persistencia de elementos vegetales concretos. Así, una de las conclusiones coincidentes entre este grupo de estudios es justamente que aquellos habitantes con niveles de renta más altos generan áreas urbanas con 
mayor diversidad biológica que aquellos que disponen de un nivel de renta menor. Este fenómeno descrito por Hope et al. (2003) como «luxury concept» (concepto de lujo), se ajusta a la lógica del gradiente socioeconómico.

No obstante, existen limitaciones en la aplicación de los gradientes socioeconómicos en áreas urbanas gestionadas de forma pública. En cualquier caso, la aplicación de gradientes socioeconómicos se convierte en una herramienta eficiente para mejor la comprensión de los ecosistemas urbanos, ayudando así a gestionar y planear mejor nuestras ciudades.

\section{LA BIODIVERSIDAD VEGETAL EN LOS AMBIENTES URBANOS}

\section{IV.1. La influencia de la urbanización en la vegetación}

El Convenio sobre la Diversidad Biológica (Naciones Unidas, 1992) define biodiversidad como el conjunto de organismos vivos que habitan en un ecosistema, o grupos de ecosistemas, y comprende la diversidad dentro de cada especie (diversidad genética), la diversidad entre las especies (diversidad taxonómica) y la diversidad de los ecosistemas (diversidad ecológica). El proceso de urbanización ejerce influencia sobre la biodiversidad, ya sea tanto en aspectos positivos como negativos. Además, los humanos son físicamente, psicológicamente y socialmente dependientes de la diversidad de su entorno, tal y como ya se ha descrito en apartados anteriores.

Las especies presentes en áreas urbanas se originan a partir de tres mecanismos: (1) especies nativas que ya estaban presentes antes del desarrollo urbano, (2) especies nativas que, aunque no se encontraban previamente de forma natural, se han desarrollado en las nuevas condiciones urbanas, y (3) especies foráneas introducidas a través de la actividad humana (McKinney, 2006; Williams et al., 2009; Boada y Sànchez, 2011; 2012). Ahora bien, no todas estas especies acaban adaptándose eficientemente a este nuevo medio.

Un marco de trabajo propuesto por Williams et al. (2009) presenta cuatro tipos de filtro según la presión de selección que, de forma conjunta, determinan cuáles son las especies que prevalecen en un ambiente determinado (Figura 2). Cada uno de estos filtros puede comportar ganancias o pérdidas en la flora y la fauna de una región. Sin embargo, identificar una sola fuerza motriz que genere las variaciones en las especies es complejo, ya que diferentes filtros pueden actuar simultáneamente. No deben obviarse tampoco las influencias que el medio natural ejerce sobre la vegetación de los espacios urbanos y que pueden quedar excluidas de este sistema de filtrado (Williams et al., 2009).

El primero de los filtros hace referencia a la transformación del hábitat para explicar que cierto número de especies han sido incapaces de persistir en ecosistemas urbanos una vez su área de distribución original se ha visto reducida como consecuencia del desarrollo urbano. Este filtro, causa, en general, una pérdida neta de especies aunque sus efectos pueden ser más o menos intensos según el grado de urbanización que se haya llevado a cabo. La existencia previa de espacios de cultivo influye también en este filtro, ya que la agricultura habría causado previamente un descenso en el número y abundancia de especies respecto al hábitat natural original.

En segundo lugar, la fragmentación de los hábitats actúa también como filtro sobre la biodiversidad ya que distintos grupos de especies requieren de amplias áreas de distribución 
para que sus metapoblaciones puedan persistir a largo plazo. Muy a menudo estas grandes áreas no se encuentran en áreas urbanas donde el paisaje no construido suele encontrarse fragmentado o vagamente conectado a través de zonas de sumidero. Este filtro causa una pérdida neta de especies ya que sólo aquellos taxones adaptados a persistir en poblaciones pequeñas alcanzan la supervivencia con éxito.

En tercera instancia, los efectos urbanos sobre el medio juegan también un papel destacado en el filtrado de la biodiversidad, ya que las áreas verdes urbanas están sujetas a efectos ambientales que no están presentes, o lo están en menor importancia, en otros ecosistemas menos fragmentados (Grimm et al., 2008). Esto incluye altos niveles de contaminación atmosférica y del suelo, elevadas temperaturas por el efecto UHI (Urban Heat Island, explicado en IV.2), o el aumento del estrés hídrico, entre otros (Pickett et al., 2001, 2011; Grimm et al., 2008). Todos estos efectos, sumados, determinan la ocurrencia de determinadas especies en los nuevos hábitats antropogénicos. A la vez, también son responsables de las ganancias o pérdidas de taxones en los fragmentos de espacio natural aislados dentro de la matriz urbana. Este tipo de filtro selecciona sólo las especies adaptadas a las perturbaciones urbanas (Williams et al., 2009). Estas perturbaciones suelen tener un carácter permanente, inhibiendo así el proceso de sucesión biológica (Cilliers y Siebert, 2010).

Figura 2

DIAGRAMA DE LOS CUATRO MAYORES FILTROS URBANOS QUE DETERMINAN LOS FLUJOS DE BIODIVERSIDAD VEGETAL EN AMBIENTES URBANOS

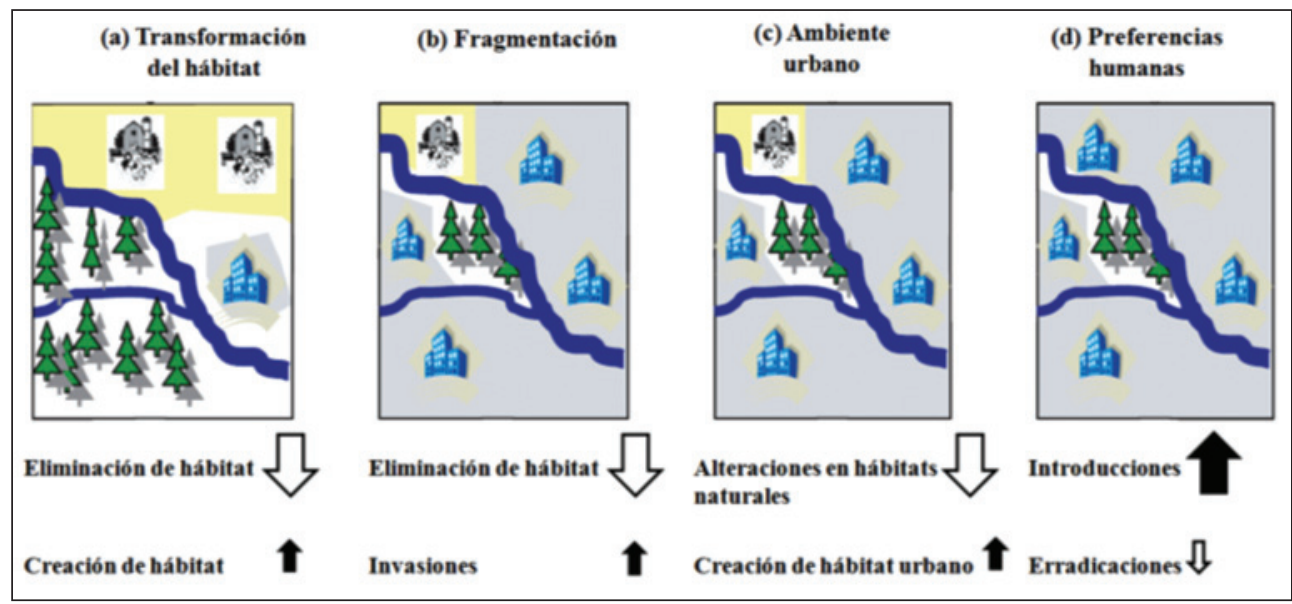

Fuente: Elaborado a partir de Williams et al., 2009. Las áreas urbanas (icono de edificios) se pueden desarrollar ya sea a partir de vegetación nativa (icono de árbol) o tierra agrícola (icono de granja). Las flechas negras representan ganancia de especies y las blancas perdida de especies. Su tamaño es proporcional a la predominancia del fenómeno dentro del filtro.

Por último, y en cuarto lugar, también las preferencias humanas se apuntan como agente influyente sobre los flujos de biodiversidad en ambientes urbanos. De este modo, la composición florística de los hábitats antropogénicos responde en gran medida a la combinación de dos fenómenos de incorporación de especies: las plantaciones con fines hortícolas y el 
desarrollo de plantas exóticas adventicias, es decir, aquellas plantas que aparecen en dichos ecosistemas de forma no deseada como podrían ser algunas especies del género Amaranthus o Chenopodium. (Williams et al., 2009). Las preferencias humanas ejercen una fuerte presión de selección sobre el número y el tipo de especies exóticas introducidas en los hábitats urbanos, así como también en la forma en que éstas son gestionadas (Hope et al., 2003; Luck et al., 2009). La probabilidad de que se establezca una nueva población se encuentra directamente relacionada con la incorporación del número de individuos con capacidad reproductiva. En este sentido, la preferencia humana actúa como filtro evidente favoreciendo algunas especies por encima de otras. Williams et al. (2009) concluye que tales preferencias comportan un aumento neto del número de especies del sistema.

Existen varios estudios que confirman todo lo que se ha discutido sobre los efectos de la urbanización y la presión en el filtrado de especies (Sax y Gaines, 2003; McKinney, 2008). En todos ellos, se concluye que las áreas urbanas, en general, disponen de un mayor número de especies que las áreas naturales y agrícolas adyacentes. Es decir, la diversidad gamma, que equivale al número total de especies entre hábitats conectados, es mayor en áreas urbanas que en otros ecosistemas contiguos. En este contexto, las áreas periféricas albergan mayor biodiversidad que las zonas urbanas más céntricas (Rueda, 1995). McKinney (2006), por su parte, llega a la conclusión que la vegetación entre ciudades es más parecida entre sí que la vegetación de diferentes áreas naturales comparadas entre ellas. En otros términos, las áreas urbanas disponen de una menor diversidad beta (la presente entre hábitats de un mismo ecosistema) que las áreas naturales. Así pues, aunque la biodiversidad local pueda verse ampliada por la llegada de especies vegetales exóticas, la biodiversidad nativa tiende a descender, conduciendo así la situación general hacia un estadio de «homogeneización biótica» (Sax y Gaines, 2003). En este sentido, Boada y Sànchez (2011; 2012) apuntan que existen dos estrategias para favorecer la biodiversidad urbana: por una parte la naturación (estrategias para incrementar el verde urbano sostenible) y por otra la naturalización (proceso de facilitación de entrada de la biodiversidad faunística en base a la naturación).

\section{IV.2. La importancia de la biodiversidad vegetal urbana como productora de bienes y servicios}

La diversidad de flora y fauna en los ecosistemas urbanos juega un papel esencial en la generación de bienes y servicios ambientales a la comunidad. Estos aprovechamientos se definen como los «beneficios que la población humana obtiene, directa o indirectamente, de las funciones de los ecosistemas» (Costanza et al., 1997: 1). Los bienes producidos por estos espacios verdes pueden ser alimentos, combustible, fibras, o productos farmacéuticos e industriales, entre otros. Por su parte, los servicios que ofrecen los ecosistemas pueden ser el ocio, la educación, la filtración del aire, la reducción del ruido, o la prevención de la erosión por parte del escurrimiento de aguas superficiales (Cameron et al., 2012).

Las grandes áreas metropolitanas suelen alcanzar temperaturas más elevadas que las áreas rurales adyacentes a ellas. Este fenómeno, descrito por Howard (1818-1820) es el llamado efecto «isla de calor urbana», y proviene del término anglosajón «Urban Heat Island» (UHI). La relación entre el espacio verde urbano y la mitigación del efecto UHI se encuentra actualmente en fase de estudio (Alexandri y Jones, 2008). Gill et al., (2007) sugiere que un $10 \%$ en el incremento de la superficie verde urbana prevendría la subida de $4{ }^{\circ} \mathrm{C}$ prevista para 
los próximos 80 años, en la ciudad de Manchester (Inglaterra). Concretamente, la presencia de árboles en estos espacios se convierte en el factor clave en la reducción de la temperatura urbana, gracias en parte a la sombra que confieren pero también a su elevada evapotranspiración (Akbari et al., 1997). Además, los árboles juegan simultáneamente un papel importante en la filtración del aire y la fijación de dióxido de carbono (Bolund y Hunhammar, 1999). Ahora bien, más allá de la influencia de los árboles, un amplio rango de vegetación dispone de potencial para enfriar el espacio urbano, aunque será su situación la que tendrá consecuencias más significativas sobre la regulación térmica. Estudios realizados en el continente americano sugieren que la localización estratégica de las plantas en espacios urbanos puede reducir entre un 20\% - 40\% el consumo energético de los edificios (Akbari et al., 1997, 2001). A pesar del innegable potencial que tiene la vegetación urbana para paliar el efecto UHI, su efectividad queda determinada por la disponibilidad de agua. Así, por ejemplo, el enfriamiento potencial al alcance de un espacio ajardinado constituido de césped está fuertemente relacionado con el riego que se le destina (McPherson et al., 1989). De forma general, quedan aún varios aspectos por resolver como la manera en que se integran y se comparan los parámetros climáticos y la vegetación a diferentes escalas (Stewart, 2011).

El balance de carbono es también un elemento destacable en el conjunto de servicios de los ecosistemas. Las plantas leñosas disponen de una capacidad de fijación de carbono más amplia que las plantas anuales debido a la capacidad de almacenamiento que tiene su biomasa (Jo y McPherson, 2002). Así, en los jardines domésticos puede llegar a almacenarse un promedio de $2,5 \times 10^{3} \mathrm{~g} / \mathrm{m}^{2}$ de carbono, teniendo en cuenta que un $83 \%$ se encuentra en el suelo, un $16 \%$ en árboles y arbustos y sólo un $0,6 \%$ en las especies vegetales herbáceas (Jo y McPherson, 2002). Las prácticas de jardinería con actitudes responsables como la reducción de herbicidas, el uso de agua reciclada, o disponer de una vegetación heterogénea son también factores que condicionan las emisiones de $\mathrm{CO}_{2}$ y que por tanto juegan un papel destacado en el balance global de producción de este gas (Pouy et al., 2002).

Algunas plantas, especialmente árboles y arbustos, pueden liberar compuestos biogenéticos orgánicos volátiles (BVOCs), que tienen un elevado potencial para producir ozono cuando reaccionan con óxidos de nitrógeno resultantes de las actividades humanas (Benjamin y Winer, 1997). Las cantidades y características específicas de los BVOCs son particulares para cada especie, y hay que tenerlo en cuenta para utilizar las especies más adecuadas en las plantaciones y en los planeamientos urbanos (Paoletti, 2009). De forma general, hay un gran nivel de incertidumbre sobre el rol de la vegetación urbana en la eliminación de estos contaminantes (Pataki et al., 2011).

El agua, entendida como recurso, también puede experimentar variaciones en sus ciclos de entrada y salida, y convertirse en un factor limitante en el mantenimiento de la biota o conjunto de organismos vivos de los espacios urbanos. Las zonas verdes ornamentales se encuentran a menudo asociadas a un elevado consumo de agua, sobre todo en periodos de sequía cuando éstos requieren de una mayor aportación hídrica. Este elevado requerimiento de agua puede, además, limitar el acceso al recurso por parte de los organismos que no disponen de una aportación artificial. En espacios privados, especialmente en urbanizaciones con viviendas unifamiliares con jardín, el consumo de agua para riego representa una parte substancial del total de agua doméstica utilizada, pudiendo suponer entre el $30 \%$ y el $70 \%$ del agua total consumida en los hogares (Domene y Saurí, 2003; Salvador et al., 2011). En 
este contexto de demanda hídrica global, hay que añadir, además, los efectos que durante los próximos años pueda tener el cambio climático sobre aquellas regiones más sensibles a las variaciones térmicas y pluviométricas como es la región mediterránea (Sala et al., 2000; Ribas et al., 2010).

Los ecosistemas urbanos desarrollan también una función en la regulación de la escorrentía de las aguas superficiales y las inundaciones asociadas. La vegetación, especialmente los árboles, interceptan el agua de la precipitación y la mantienen temporalmente en la superficie de su dosel reduciendo así su flujo hacia el suelo (Xiao y McPherson, 2002). Además, la vegetación también mitiga el efecto de las inundaciones al aumentar la infiltración a través del suelo (Dunne et al., 1991). Sin embargo, la tendencia general en diversas áreas urbanas está comportando un aumento de la superficie pavimentada artificialmente (García, 2012). En este sentido, Pauleit y Duhme (2000) comprobaron que los espacios urbanos de baja densidad estaban vinculados a episodios de escorrentía e inundaciones menos severos que aquellos espacios de mayor densidad. Por este motivo, en Inglaterra, y desde 2008, es necesario un permiso para cambiar el pavimento vegetal por pavimento artificial en los hogares (Anon, 2009).

Según Chiesura (2004), el espacio verde de las ciudades también se usa por diferentes motivos como la relajación, la liberación del estrés de la ciudad, la vivencia de sensaciones positivas de contacto con la naturaleza, entre otros. Estas experiencias tienen consecuencias tanto a nivel físico, psicológico como social, influenciando así el bienestar y la calidad de vida de los ciudadanos (Clayton, 2007). Así, por ejemplo, algunas investigaciones han demostrado que los pacientes expuestos a ambientes naturales durante su proceso de recuperación se recuperan más rápido que aquellos que lo hacen en ambientes más edificados y construidos (Ulrich, 1981).

Finalmente, los espacios verdes urbanos, pero también periurbanos, suponen también una oportunidad para incrementar la agricultura de subsistencia (Domene y Saurí, 2007). Actualmente, una séptima parte del alimento de nuestro planeta es producida a través de la agricultura urbana, incluyendo también aquella que tiene lugar en jardines domésticos (Olivier, 1999). Este hecho es especialmente importante en comunidades pobres de países en vías de desarrollo (Shackleton et al., 2008), pero también en muchos otros países. En Catalunya, donde la presencia de huertos en espacios residenciales es cada vez mayor, estos pueden llegar a representar hasta un 6,5\% del total de la superficie exterior en áreas residenciales (García, 2012).

\section{EL CASO PARTICULAR DE LOS JARDINES DOMÉSTICOS URBANOS}

\section{V.1. Breve historia de los jardines domésticos contemporáneos}

La especie humana ha utilizado la horticultura, es decir, el cultivo y cuidado de las plantas, desde hace más de 3000 años (Hadidi, 1984). A pesar de que en sus inicios los propósitos de cultivo eran puramente utilitarios, algunas culturas más desarrolladas, como la romana, comenzaron a cultivar las plantas para adornar estéticamente sus viviendas y les otorgaron distintos valores (Guillot, 2009). Además, estos espacios jugaban un papel simbólico en la concepción del «Paraíso» y estaban cargados de elementos espirituales (Bennis, 2006). Así, los romanos dividían el espacio ajardinado de sus hogares en cuatro partes donde plantaban 
diferentes especies vegetales: hortalizas, flores, plantas aromáticas o medicinales, árboles frutales y cipreses, este último vinculado al simbolismo espiritual (Guillot, 2009). Ahora bien, la jardinería ornamental, tal y como la conocemos hoy, se originó a partir del siglo XII y XIII (Owen, 1991), y fue la expansión colonial, junto con el descubrimiento de nuevas partes del mundo, las que aportaron nuevas especies en el comercio mundial de este tipo de plantas (Reichard y White, 2001).

Durante la Edad Moderna, y gran parte de la contemporánea, los jardines eran diseñados mayoritariamente por arquitectos que daban respuesta a los cánones estéticos, culturales e ideológicos de cada época en concreto. A menudo estos espacios se ubicaban en grandes parcelas de espacio público para cumplir, además, un papel simbólico, o bien en propiedades privadas de colectivos con elevado poder adquisitivo. No será, sin embargo, hasta el siglo XX que la jardinería entrará de lleno en el planeamiento urbano de las ciudades.

En las últimas décadas, determinadas zonas urbanas del litoral Mediterráneo han experimentado una reestructuración en forma de proceso de dispersión (Dura-Guimera, 2003; Muñoz, 2003). Este proceso a menudo ha comportado un desarrollo urbano de baja densidad con modelos de ciudad difusa y con características típicas de los patrones urbanos anglosajones, lo cual difumina aún más la identidad propia de cada ciudad (Rueda, 1995; Muñoz, 2007). Esta explosión urbanística laxa, ha conllevado inevitablemente un aumento del número de jardines domésticos privados. En gran medida, estos espacios han tendido a ocupar superficies relativamente pequeñas de territorio, pero su elevada proliferación, especialmente en áreas residenciales, ha supuesto grandes consumos de suelo urbano a gran escala.

Los criterios utilizados para diseñar, crear y gestionar este volumen importante de jardines responden a diversas variables como las características urbanas de cada lugar, el nivel socioeconómico de sus ocupantes o incluso cuestiones psicológicas y de comportamiento, entre otras (Hope et al. 2003: Cook et al., 2012). También los patrones histórico-culturales han tenido su influencia en la forma y composición de los jardines domésticos actuales, y esto se detecta en una tendencia generalizada que parece conducir los jardines hacia una globalización de la flora urbana (Faggin y Ignatieva, 2009). Así pues, crece la importancia de preservar el patrimonio local, natural y cultural, para crear ciudades únicas con ecosistemas particulares y eficientes al mismo tiempo.

\section{V.2. Biodiversidad vegetal en jardines domésticos como parte del verde urbano}

Los jardines domésticos, en general, son gestionados de forma privada. Este atributo ha comportado que a menudo estos espacios quedaran excluidos del balance global de espacios verdes presentes en las ciudades, conllevando así un sesgo sustancial respecto al total de zonas verdes reales (Gaston et al., 2005). En Inglaterra, se calcula que los jardines domésticos constituyen prácticamente una cuarta parte de la superficie total de cinco de las ciudades más pobladas del país (Gaston et al., 2005; Loram et al., 2007). En la ciudad de Dunedin (Nueva Zelanda), el porcentaje podría variar hasta el 36\% del total del área urbana (Mathieu et al., 2007), y en Baltimore (Michigan), hasta el $90 \%$ del dosel de los árboles se encuentra en espacios privados (Troy et al., 2007). Por lo tanto, aunque los jardines, de forma individual, suelen representar una parte relativamente pequeña del territorio, cuando se consideran como conjunto abarcan gran parte de las superficies urbanas totales. 
Más allá de los bienes y servicios que los jardines domésticos puedan aportar, ya sea a la sociedad o al ecosistema en general, estos espacios reúnen una diversidad biológica considerable y superior a la mayoría de espacios urbanos más cercanos (Thompson et al., 2003), y por lo tanto su importancia ecológica debe ser considerada en cualquier toma de decisiones (Terradas, 2001). El mantenimiento de estas estructuras semi-naturales conlleva, además, la inversión de capital económico por parte de los propietarios, y por tanto la industria de la horticultura juega un papel importante en la promoción de la biodiversidad y de las medidas de gestión y ahorro adecuadas (Lubbe, 2011).

Thompson et al. (2003) proponen dos motivos por los cuales los jardines domésticos presentan una variedad tan grande de especies vegetales: (1) la gran oferta de plantas disponibles a la venta, y (2) el significativo esfuerzo de mantenimiento de estos ecosistemas por parte de propietarios, y jardineros especializados. Este esfuerzo sin duda dota a las especies de la habilidad antinatural de persistir con un número escaso de individuos. En cuanto a la primera de las razones, algunos estudios han aportado datos sobre la amplia variedad de plantas a la venta con fines ornamentales. Así, en Inglaterra se pueden adquirir un total de 70.000 taxones (Macaulay et al., 2009), y en Estados Unidos 90.000 (Isaacson, 2004). En España, la obra «Flora Ornamental Española» de Sánchez et al. (2000-2010), aunque se encuentra inacabada, prevé la descripción de más de 11.000 taxones empleadas en jardinería ornamental. Esta cifra supera ampliamente los 8.300 taxones descritos en el conjunto de la flora española (Blanco, 1988). Así pues, ante esta gran disponibilidad de plantas, y añadiendo que regularmente se incorporan nuevas especies a la oferta, la variedad de taxones al alcance de la jardinería es muy extensa. Asociado a este hecho se encuentra el aumento de especies exóticas que son usadas regularmente con fines ornamentales. Así, varios estudios han analizado el porcentaje de especies exóticas presentes en los jardines domésticos: 88\% en la región de Lauris (Francia) (Marco et al., 2008), 85\% en Bujumbura (Burundi) (Bigirimana et al., 2012), o 75\% en Trabzon (Turquía) (Acar et al., 2007).

Esta proporción de especies exóticas puede poner en peligro la vegetación vulnerable de los espacios naturales adyacentes en caso de que las especies puedan naturalizarse y convertirse en invasoras (Dehnen-Schmutz et al., 2007). En Alemania, se calcula que el 50\% de flora invasora fue introducida de forma deliberada, y más de la mitad de esta tiene un origen ornamental (Kühn y Klotz, 2006). Por su parte, en la República Checa, el 53\% de la flora introducida deliberadamente tiene el mismo origen ornamental (Pyšek et al., 2002), y en Australia el 65\% de las plantas establecidas entre 1971 y 1995 fueron introducidas con estos mismos propósitos (Groves, 1998). En España, Sanz-Elorza et al., (2004) calculan que aproximadamente un $12 \%$ del total de la flora del país está constituida por flora exótica, y un $48 \%$ de ésta ha tenido la horticultura y la jardinería como causa de introducción principal. En este sentido, la gestión incontrolada de los residuos de jardinería puede actuar como un foco muy eficiente de dispersión (Sullivan et al., 2005).

\section{V.3. El jardín doméstico y su papel en la conservación biológica y sensibilización ambiental}

A pesar de la creciente toma de conciencia del potencial de los jardines privados como herramienta de conservación, pocas investigaciones ecológicas se han ocupado de eva- 
luar el estado de la vida salvaje de estos ambientes. Este hecho se debe, principalmente, a que estos jardines son vistos como ecosistemas débiles donde el acceso es complicado debido a su marcado carácter privado. La mayoría de estudios en jardines domésticos privados se han llevado a cabo en países desarrollados y tomando un solo jardín como elemento de estudio para analizar sus características a lo largo de un período prolongado de tiempo (Owen, 1991). Los estudios a corto plazo de varios jardines domésticos están tomando fuerza en diversos centros de investigación, destacando notablemente el proyecto BUGS (Biodiversity in Urban Gardens in Sheffield), que incluye el muestreo de la flora y la fauna de más de 300 jardines de diferentes ciudades de Inglaterra (ej. Gaston et al., 2005; Smith et al., 2006; Loram et al., 2008). Estudios similares se han realizado en Norte América (Fetridge et al., 2008), resto de Europa (Marco et al., 2008), Asia (Acar et al., 2007; Jaganmohan et al., 2012), o África (Lubbe, 2011; Bigirimana et al., 2012). En países en vías de desarrollo destaca el papel del «huerto familiar» («homegarden» en términos anglosajones) para el sustento económico y alimenticio de los hogares (Fernandes y Nair, 1986). Esta tipología de jardín posee funciones distintas respeto a los jardines urbanos en países desarrollados y albergan altos niveles de biodiversidad singular especialmente en áreas tropicales (Kumar y Nair, 2004).

Por otra parte, la protección de la flora de los jardines es importante ya que proporciona hábitat y alimento a diferentes especies (Kendle y Forbes, 1997). La gran riqueza de especies, junto con la gran extensión que pueden llegar tener las áreas ajardinadas, proporciona muchas oportunidades de conservación en diferentes lugares. La conservación de estos espacios privados queda, en gran parte, fuera del alcance de las administraciones públicas, y por tanto los recursos que se pueden destinar para este propósito son muy limitados. Varios países, como Inglaterra o Estados Unidos, cuentan con organizaciones y ONGs que promueven estrategias denominadas «wildlife-friendly» para acercar la importancia ecológica de los jardines sobre una opinión pública muy a menudo escéptica hacia la conservación de estos espacios (Goddard et al., 2009). Además, algunos gobiernos en países como Australia e Inglaterra, han publicado documentos para animar a la población civil a participar en la preservación de su espacio natural más próximo, favoreciendo así la conservación de la naturaleza urbana (Goddard et al., 2009).

El esfuerzo de conservación tiene como elemento fundamental la valoración que el ser humano hace del espacio natural que le rodea (Kendle y Forbes, 1997; Miller, 2005). Ahora bien, este fenómeno está menguando con el paso del tiempo bajo un proceso llamado «environmental generational amnesia» (Kahn, 2002). Este proceso describe como cada nueva generación tiene menos sensibilidad ambiental que la generación precedente debido a una progresiva disminución del contacto directo con la naturaleza en una época plenamente urbana.

Teniendo en cuenta estos planteamientos, el eje central de la conservación en espacios urbanos debe centrarse en mejorar la calidad de vida de sus habitantes más que en la conservación entendida como tal (Miller, 2005). No obstante, cada tipología de área verde urbana debe ser analizada por separado para evaluar su papel en la conservación de la biodiversidad (Terradas, 2001). Para conseguir estos propósitos se dispone de herramientas como la educación ambiental, el uso de un lenguaje comprensivo para comunicarse con los gestores, la implicación de diferentes actores del territorio (Miller y Hobbs, 2002), o la inclusión de dis- 
ciplinas de diferentes ámbitos que permitan una visión más amplia de conceptos como «biodiversidad» o «conservación» (Cilliers et al., 2004). En este sentido, los jardines domésticos sirven de punto de contacto entre el espacio natural y el espacio urbano, y también como instrumento para acercar los valores ecológicos y sociales de los ecosistemas a la sociedad. Por lo tanto, el aprovechamiento de estos espacios debe favorecer una toma de conciencia global hacia la protección del medio natural.

\section{CONCLUSIONES}

En una época de crecimiento urbano global, la necesidad de desarrollar una ecología urbana que permita entender mejor la relación entre la sociedad y los ecosistemas naturales cobra cada día más importancia. La revisión presentada pretende profundizar en algunos aspectos relevantes de esta disciplina en relación al estudio de la biodiversidad vegetal en zonas verdes urbanas.

De este modo, la ecología urbana se ha descrito como una ciencia emergente que recoge el factor antrópico como principal agente de cambio de los ecosistemas urbanos. Su interdisciplinariedad permite el uso de técnicas y principios de disciplinas como la ecología del paisaje, la biología, la geografía e incluso la economía o el urbanismo. En concreto, y para el estudio de la flora urbana, se propone un marco trabajo preliminar que recoge de forma sintética gran parte de los conceptos revisados en este estudio (Figura 3).

En primera instancia, y como paso previo, es indispensable seleccionar el tipo de zona verde (o zonas) que se pretende estudiar de acuerdo con los objetivos planteados. De este modo se da paso al segundo estadio en que el investigador analiza cuales son las disciplinas y metodologías que más se ajustan a los propósitos de su estudio. Este acercamiento inicial debe ser transversal para captar información relevante de distintos ámbitos no estrictamente relacionados con la flora urbana. Solo así se consigue consolidar una ecología urbana adaptada a los propósitos de cada investigación.

En la tercera fase es esencial un ejercicio de descripción de los términos y conceptos con los que se pretende trabajar (por ejemplo: urbano, natural, zona verde, jardín doméstico). La falta de precisión con la que a menudo son tratados, y la falta de fronteras claras entre ellos, puede conllevar interpretaciones sesgadas. Además, de este modo se asegura la reproducibilidad de métodos a escala global. Por otra parte, la descripción del tipo de vegetación que se pretende analizar (cultivada, espontánea, solo árboles, etc.) es también fundamental para afinar en la descripción del estudio. En este punto, se recomienda retroceder hasta la fase anterior para revisar de nuevo la información de base y completarla si fuese necesario. De este modo el concepto de ecología urbana utilizado en cada enfoque evoluciona a lo largo del proceso y se consigue una visión más integrada de la realidad territorial.

Una vez delimitada la unidad de estudio, su contexto territorial y el marco teórico, la fase cuatro prevé el uso de distintos métodos adaptados a la escala de trabajo. La clasificación de la matriz urbana, por un lado, permite la descomposición del mosaico urbano en fragmentos que serán analizados a través de índices del paisaje. Así, la estructura vegetal y la estructura urbana se interrelacionan dentro de una misma dinámica espacial. Por su parte, y a una escala mayor, el estudio de los patrones en forma de gradientes permite analizar a gran detalle la respuesta de las comunidades vegetales frente a cambios ambientales y socioeconómicos 
graduales. Ambas aproximaciones pueden complementarse entre sí enriqueciendo la calidad de los resultados. Aunque en la Figura 3 solo se detallan estos dos métodos, no se descartan otras aportaciones metodológicas.

Figura 3

PROPUESTA DE MARCO DE TRABAJO GENERAL PARAAPROXIMACIÓN A LA BIODIVERSIDAD VEGETAL URBANAA TRAVÉS DE LA ECOLOGÍA URBANA

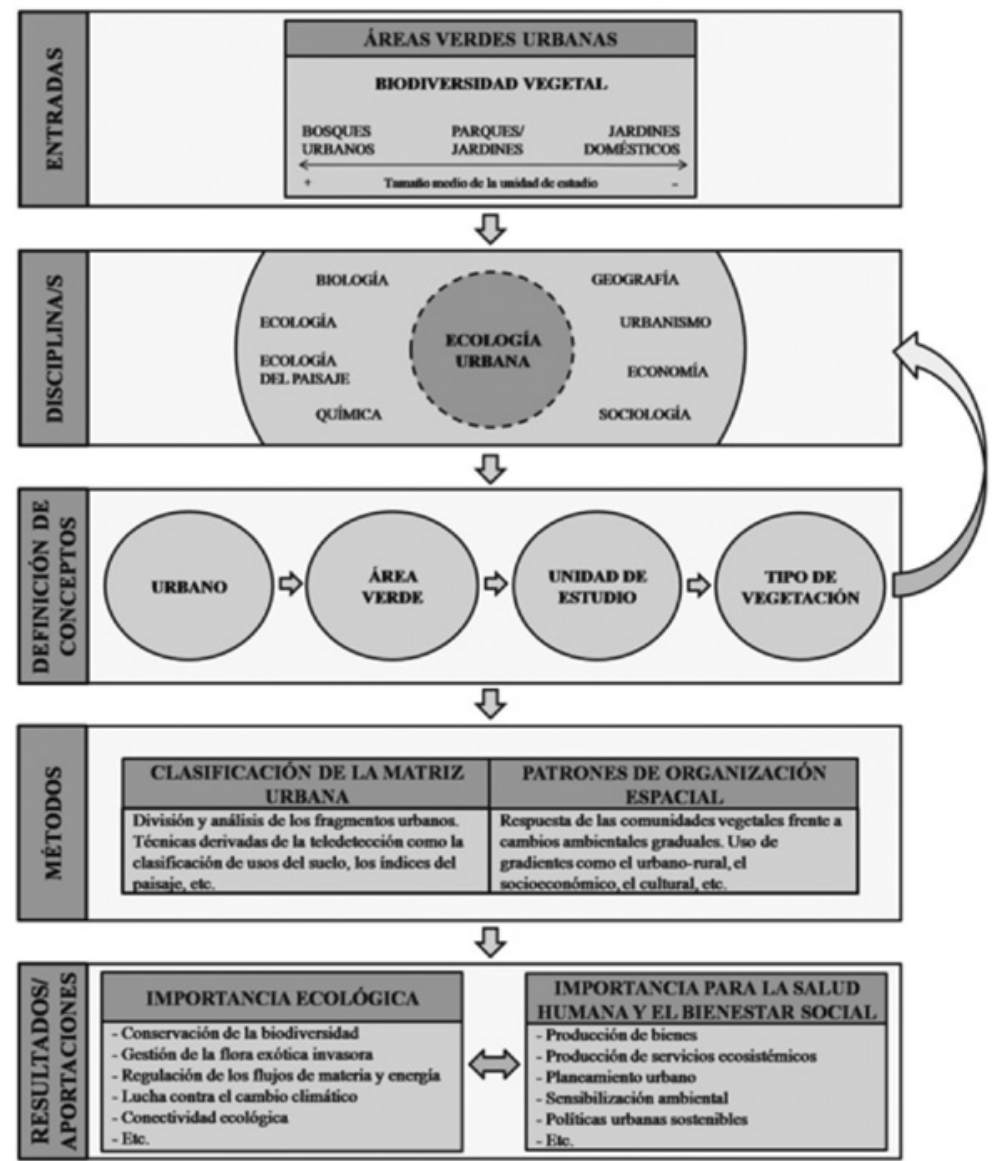

Fuente: elaboración propia.

Finalmente, en la última fase, los resultados y aportaciones de todo el proceso se clasifican según su importancia ecológica y también su importancia para la salud humana y el bienestar social. En este sentido, la vegetación urbana en general, y por lo tanto también aquella presente en jardines domésticos, puede contribuir a la conservación y protección de diversos recursos y valores naturales. Las ciudades contienen un mayor número de especies que otras áreas cercanas a ellas en gran parte gracias a la llegada de especies exóticas. Al mismo tiempo, esto conlleva procesos de homogeneización biótica entre zonas urbanas y 
otras problemáticas asociadas a las invasiones biológicas. Por otra parte, las zonas verdes urbanas, públicas y privadas, pueden contribuir como elemento de sensibilización ambiental y acercamiento de los valores de los ecosistemas naturales. Sin duda este es un punto clave para incorporar en el planeamiento urbano, el cual debe favorecer medidas que refuercen los vínculos entre la población urbana y el medio natural. Así, si los procesos de urbanización condicionan la biodiversidad y esta, a su vez, influye en el bienestar social, el planeamiento urbano tiene el reto de mejorar la calidad de vida en las ciudades.

Los estudios sobre biodiversidad vegetal urbana desde el punto de vista de la ecología urbana han abordado en los últimos años diferentes temáticas como la fijación de gases, la escorrentía superficial, la contaminación por pesticidas, la conservación biológica, etc. Distintos autores destacan el uso de gradientes como metodología para analizar la distribución y dinámica de la flora urbana. En el caso concreto de jardines domésticos, su carácter privado constituye una oportunidad excelente para ponerlo en práctica y evaluar las interacciones entre los factores socioeconómicos y culturales en relación con la composición de la flora urbana. Es justo en este tipo de ambientes, donde las preferencias humanas y el sustrato cultural tienen una mayor interacción con las dinámicas vegetales. No obstante, en el ámbito español existe poca literatura de referencia en la materia que utilice este tipo de aproximación.

En definitiva, la información presentada refleja la importancia de las áreas verdes urbanas y la ecología urbana en un mundo cada vez más poblado. Esta nueva disciplina se presenta como una ciencia interdisciplinaria que debe ser incorporada por ecólogos y planificadores en futuros estudios y no debe ser descuidada en un contexto de cambio global que sin duda marcará las tendencias de los próximos tiempos.

\section{BIBLIOGRAFÍA}

ACAR, C., ACAR, H. y EROGLU, E. (2007): «Evaluation of ornamental plant resources to urban biodiversity and cultural changing: a case study of residential landscapes in Trabzon City (Turkey)». Building and Environment, $\mathrm{n}^{\circ} 42,218-229$.

ADAMS, C.C. (1935): «The relation of general ecology to human ecology». Ecology, n' 16, 316-335.

AKBARI, H., KURN, D.M., BRETZ, S.E. y HANFORD, J.W. (1997): «Peak power and cooling energy savings of shade trees». Energy and Buildings, $\mathrm{n}^{\circ} 25,139-148$.

AKBARI, H., POMERANTZ, M. y TAHA, H. (2001): «Cool surfaces and shade trees to reduce energy use and improve air quality in urban areas». Solar Energy, n 70, 295-310.

ALBERTI, M. (2005): «The effects of urban patterns on ecosystem function». International Regional Science Review, $\mathrm{n}^{\circ} 28,168-192$.

ALBERTI, M., BOTSFORD, E. y COHEN, A. (2001): «Quantifying the urban gradient: linking urban planning and ecology» en Avian ecology and conservation in an urbanizing world (Marzluff, J.M., Bowman, R. y Donnelly, R., coord.). Norwell, MA, Kluwer Academic Publishers, 89-115.

ALBERTI, M., MARZLUFF, J.M., SHULENBERGER, E., BRADLEY, G., RYAN, C. y ZUMBRUNNEN, C. (2003): «Integrating humans into ecology: opportunities and challenges for studying urban ecosystems». BioScience, $\mathrm{n}^{\circ}$ 53, 1169-1179. 
ALEXANDRI, E. y JONES, P. (2008): «Temperature decreases in an urban canyon due to green walls and green roofs in diverse climates». Building and Environment, ${ }^{\circ}$ 43, 480493.

ANON, (2009): Guidance on the Permeable Surfacing of Front Gardens. London. Department for Communities and Local Government, 1-28.

BARRACÓ, H., PARÉS M., PRAT, A. y TERRADAS, J. (1999): Barcelona 1985-1999. Ecologia d'una ciutat. Barcelona. Ajuntament de Barcelona.

BENJAMIN, M.T. y WINER, A.M. (1997): «Estimating the ozone-forming potential of urban trees and shrubs». Atmospheric Environment, $\mathrm{n}^{\circ} 32$ (1), 53-68.

BENNIS, E. (2006): The Story of Gardens in Europe: Manchester Metropolitan University, EGHN.

BIGIRIMANA, J., BOGAERT, J., DE CANNIÈRE, C., BIGENDAKO, M-J. y PARMENTIER, I. (2012): «Domestic garden plant diversity in Bujumbura, Burundi: Role of the socio-economical status of the neighborhood and alien species invasion risk». Landscape and Urban Planning, $\mathrm{n}^{\circ}$ 107, 118-126.

BINO, G., LEVIN, N., DARAWSHI, S., VAN DER HAL, N., REICH-SOLOMON, A. y KARK, S. (2008): «Accurate prediction of bird species richness patterns in an urban environment using Landsat-derived NDVI and spectral unmixing». International Journal of Remote Sensing, $\mathrm{n}^{\circ} 29,3675-3700$.

BLANCO, E. (1988): «Nuestra flora en peligro». Vida Silvestre, n 63, 2-13.

BOADA, M. y SÀNCHEZ, S. (2011): «Natura i ciutat, biodiversitat urbana» en Cap a un habitatge sostenible. (Informes del CADS: 12) (Gausa, M. coord.). Barcelona, Consell Assessor per al Desenvolupament Sostenible, Departament de la Presidència, Generalitat de Catalunya, 107-112.

BOADA, M. y SÀNCHEZ, S. (2012): «Naturaleza y cultura, biodiversidad urbana» en Ecoinovaçao para a melhoria ambiental de produtos e serviços. Experiências espanholas e brasileiras nos setores industrial, urbano e agrícola (Ometto, A.R., Bovo, R. y Saavedra, Y.M.B., coord.). Sao Carlos, Diagrama Editorial, 131-142.

BOLUND, P. y HUNHAMMAR, S. (1999): «Ecosystem services in urban areas». Ecological Economics, $\mathrm{n}^{\circ}$ 29, 293-310.

BUJÁN, M., DÍAZ-VIZCAÍNO, E., CASCUDO, A., IGLESIAS, A. y RIGUEIRO, A. (1998): «Competition floristique et abondance des mauvises herbes des anciens remparts de Lugo (Galice, Espagne)» en Proceedings 6ème Symposium Mediterraneen EWRS. Montpellier, 221-223.

CADENASSO, M.L., PICKETT, S.T.A. y SCHWARZ K. (2007): «Spatial heterogeneity in urban ecosystems: reconceptualizing land cover and a framework for classification». Frontiers in Ecology and the Environment, $\mathrm{n}^{\circ}$ 5, 80-88.

CAMERON, R.W.F., BLANUSA, T., TAYLOR, E. J., SALISBURY, A., HALSTEAD, A.J., HENRICOT, B., y THOMPSON, K. (2012): «The domestic garden - Its contribution to urban green infrastructure». Urban Forestry \& Urban Greening, n 11, 129-137.

CHIESURA, A. (2004): «The role of urban parks for the sustainable city». Landscape and Urban Planning, ${ }^{\circ}$ 68, 129-138.

CHIVIAN, E. y BERNSTEIN, A. (2008): Sustaining life: how human health depends on biodiversity. New York. Oxford University Press. 568 p. 
CILLIERS, S.S. (2010): «Social aspects of urban biodiversity - an overview» en Urban biodiversity \& design (Muller, N., Werner, P., Kelcey, J. coord.). Inglaterra, Blackwell Publishing, 81-100.

CILLIERS, S.S. y SIEBERT, S.J. (2010): «Urban flora and vegetation: patterns and processes» en Urban ecology: patterns, processes, and applications (Niemela, J., coord.). Oxford, Oxford University Press, 148-158.

CILLIERS, S.S., MÚLLER, N. y DREWES, E. (2004): «Overview on urban nature conservation: situation in the western-grassland biome of South Africa». Urban Forestry and Urban Greening, $\mathrm{n}^{\circ}$ 3, 49-62.

CLAYTON, S. (2007): «Domesticated nature: Motivations for gardening and perceptions of environmental impact». Journal of Environmental Psychology, $\mathrm{n}^{\circ}$ 27, 215-224.

COLDING, J. (2007): «Ecological land-use complementation for building resilience in ecosystems». Landscape and Urban Planning, $\mathrm{n}^{\circ}$ 81, 46-55.

COLLINS, J.P., KINZIG, A., GRIMM, N.B., FAGAN, W.F., HOPE, D., WU, J. y BORER, E.T. (2000): «A new urban ecology: modelling communities as integral parts of ecosystems poses special problems for the development and testing of ecological theory». American Scientist, $\mathrm{n}^{\circ} 88,416-425$.

COOK, E.M., HALL, S.J. y LARSON, K.L. (2012): «Residential landscapes as social-ecological systems: a synthesis of multi-scalar interactions between people and their home environament». Urban Ecosystems, $\mathrm{n}^{\circ} 15,19-52$.

COSTANZA, R., D’ARGE, R., DE GROOT, R., FARBER, S., GRASSO, M., HANNON, B., LIMBURG, K., NAEEM, S., O’NEILL, R., PARUELO, J., RASKIN, R., SUTTON, P. y VAN DEN BELT, M. (1997): «The value of the world's ecosystem services and natural capital». Nature, $\mathrm{n}^{\circ} 387,253-260$.

DANA, E.D., VIVAS, S. y MOTA J.F. (2002): «Urban vegetation of Almería City - a contribution to urban ecology in Spain». Landscape and Urban Planning, ${ }^{\circ}$ 59, 203-216.

DEHNEN-SCHMUTZ, K., TOUZA, J., PERRINGS, C. y WILLIAMSON, M. (2007): «The Horticultural Trade and Ornamental Plant Invasions in Britain». Conservation Biology, $n^{\circ} 21,224-231$.

DOMENE, E. y SAURÍ, D. (2003): «Modelos Urbanos y Consumo de Agua. El Riego de Jardines Privados en la Región Metropolitana de Barcelona». Investigaciones Geográficas, $\mathrm{n}^{\circ} 32,5-17$.

DOMENE, E. y SAURÍ, D. (2007): «Urbanitzation and class-produced natures: Vegetables gardens in the Barcelona Metropolitan Region». Geoforum, n 38, 287-298.

DOW, K. (2000): «Social dimensions of gradients in urban ecosystems». Urban Ecosystems, $\mathrm{n}^{\circ} 4,255-275$.

DUNNE, T., ZHANG, W. y AUBRY, B.F. (1991): «Effects of rainfall, vegetation, and microtopography on infiltration and runoff». Water Resources Research, $\mathrm{n}^{\circ} 27,2271-$ 2285.

DURA-GUIMERA, A. (2003): «Population deconcentration and social restructuring in Barcelona, a European Mediterranean city». Cities, n' 20, 387-394.

DU TOIT, M. J., CILLIERS, S. S. (2011): «Aspects influencing the selection of representative urbanization measures tot quantify urban-rural gradients». Landscape Ecology, $\mathrm{n}^{\circ}$ 26, 169-181. 
FAGGI, A. y IGNATIEVA, M. (2009): «Urban green spaces in Buenos Aires and Christchurch». Municipal Engineer, $\mathrm{n}^{\circ} 162$ (4), 241-250.

FERIA TORIBIO, J.M. y SANTIAGO RAMOS, J. (2009): «Funciones ecológicas del espacio libre y planificación territorial en ámbitos» en Scripta Nova, vol. XIII, nº 299, 15 de septiembre de 2009. Universidad de Barcelona. Disponible en http://www.ub.es/geocrit/ sn/sn-299.htm

FERNANDES, E.C.M. y NAIR, P.K.R. (1986): «An Evolution of the structure and function of tropical homegardens». Agricultural Systems, nº 21, 279-310.

FETRIDGE, E.D., ASCHER, J.S. y LANGELLOTTO, G.A. (2008): «The bee fauna of residential gardens in a suburb of New York City (Hymenoptera: Apoidea)». Annals of the Entomological Society of America, $\mathrm{n}^{\circ} 101,1067-1077$.

GASTON, K.J., WARREN, P.H., THOMPSON, K. y SMITH, R.M. (2005): «Urban domestic gardens (IV): the extent of the resource and its associated features». Biodiversity and Conservation, $\mathrm{n}^{\mathrm{o}}$ 14, 3327-3349.

GILL, S.E., HANDLEY, J.F., ENNOS, A.R. y PAULEIT, S. (2007): «Adapting cities for climate change: the role of green infrastructure». Built Environment, $\mathrm{n}^{\circ} 33,115-133$.

GODDARD, M.A., DOUGILL, A.J. y BENTON, T.G. (2009): «Scaling up from gardens: biodiversity conservation in urban environaments». Trends in Ecology and Evolution, $\mathrm{n}^{\circ}$ 25, 90-98.

GRIMM, N.B., FAETH, S.H., GOLUBIEWSKI, N.E., REDMAN, C.L., WU, J., BAI, X. y BRIGGS, J.M. (2008): «Global change and the ecology of cities». Science, $\mathrm{n}^{\circ}$ 319, 756-760.

GROVES, R. H. (1998): «Recent incursions of weeds to Australia 1971-1995». CRC for Weed Management Systems Technical Series, $\mathrm{n}^{\circ}$ 3, 1-74.

GUILLOT, D. (2009): «Flora ornamental española: aspectos históricos y principales especies». Monografías de la revista Bouteloua, $\mathrm{n}^{\circ}$ 8, $274 \mathrm{pp}$.

HADIDI, M.N.E. (1984): «Food plants of pre-historic and pre-dynastic Egypt» en Plants for arid lands (Wickins, G. E., Goodin, J.R. y Field, D.V. coord.). New York, Springer, 87-92.

HAHS, A.K. y MCDONNELL, M.J. (2006): «Selecting independent measures to quantify Melbourne's urbanrural gradient. Landscape and Urban Planning, 78(4): 435-448.

HOPE, D., GRIES, C., ZHU, W.X., FAGAN, W.F., REDMAN, C.L., GRIMM, N.B., NELSON, A.L., MARTIN, C. y KINZIG, A. (2003): «Socioeconomics drive urban plant diversity». Proceedings of the National Academy of Sciences of the United States of America, $\mathrm{n}^{\circ} 100,8788-8792$.

HOWARD, L. (1818-1820): The climate of London, deduced from Meteorological observations, made at different places in the neighbourhood of the metropolis. London. W. Phillips.

HOWES, R. y HERBERT, R. (2005): Infrastructure for the Built Environment. Oxford, Butterworth-Heinemann.

JAGANMOHAN, M., VAILSHERY, L.S., GOPAL, D. y NAGENDRA, H. (2012): «Plant diversity and distribution in urban domestic gardens and apartments in Bagalore, India». Urban Ecosystems, $\mathrm{n}^{\circ}$ 15, 911-925.

JO, H.K., y MCPHERSON, E.G. (1995): «Carbon storage and flux in urban residential greenspace». Journal of Environmental Management, $\mathrm{n}^{\circ}$ 45, 109-133. 
JOHNSON, D. D. P., HAY, S. I. y ROGERS, D. J. (1998): «Contemporary environmental correlates of endemic bird areas derived from meteorological satellite sensors». Proceedings of The Royal SocietyB $\mathrm{n}^{\mathrm{o}} 265,951-959$.

KAHN, P.H., Jr. (2002): «Children's affiliations with nature: structure, development, and the problem of generational amnesia» en Children and nature: psychological, sociocultural, and evolutionary investigations (Kahn, P.H., Jr. y Kellert, S.R. coord.). Cambridge, MA: MIT Press, 93-116.

KENDAL, D., WILLIAMS, K. J. H. y WILLIAMS N. S. G. (2012): «Plant traits link people's plant preferences to the composition of their gardens». Landscape and Urban Planning, $\mathrm{n}^{\circ} 105,34-42$.

KENDLE, T. y FORBES, S. (1997): Urban nature conservation: landscape management in the urban countryside. London. E \& FN Spon.

KINZIG, A. P., WARREN, P., MARTIN, C., HOPE, D. Y KATTI, M. (2005): «The effects of human socioeconomic status and cultural characteristics on urban patterns of Biodiversity». Ecology and Society, $\mathrm{n}^{\circ} 10(1), 23$.

KÜHN, I. y KLOTZ, S. (2006): «Urbanization and homogenization: comparing floras of urban and rural areas in Germany». Biological Conservation, ${ }^{\circ}$ 127, 292-300.

KUMAR, B.M., y NAIR, P.K.R. (2004): «The enigma of tropical homegardens». Agroforestry Systems, n ${ }^{\circ}$ 61, 135-152.

LORAM, A., THOMPSON, K., WARREN, P.H. y GASTON, K.J. (2008): «Urban domestic gadens (XII): The richness and composition of the flora in five UK cities». Journal of Vegetation Science, $\mathrm{n}^{\circ}$ 19, 321-330.

LORAM, A., TRATALOS, J., WARREN, P.H. y GASTON, K.J. (2007): «Urban domestic gardens (X): the extent \& structure of the resource in five major cities». Landscape Eco$\log y, \mathrm{n}^{\circ} 22,601-615$.

LUBBE, C.S. (2011): Comparison of the urban domestic garden flora along a socio-economic gradient in the Tlokwe City Municipality. South Africa. North-West University.

LUCK, G.W., SMALLBONE, L.T. y O'BRIEN, R. (2009): «Socio-economics and vegetation change in urban ecosystems: Patterns in space and time». Ecosystems, n $12,604-$ 620 .

LUCK, M. y WU, J. (2002): «A gradient analysis of urban landscape pattern: a case study from the Phoenix metropolitan region, Arizona, USA». Landscape Ecology, n 17, 327-339.

MARCO,A., BARTHELEMY, C., DUTOIT, T. y BERTAUDIERE-MONTES, V. (2010): «Bridging human and natural sciences for a better understanding of urban floral patterns: the role of planting practices in Mediterranean gardens». Ecology and Society, n 15(2): artículo 2. Septiembre 2010. Disponible en http://www.ecologyandsociety.org/vol15/iss2/art2/.

MARCO, A., DUTOIT, T., DESCHAMPS-COTTIN, M., MAUFFREY, J., VENNETIER, M. y BERTAUDIERE-MONTES, V. (2008): «Gardens in urbanizing rural areas reveal an unexpected floral diversity related to housing density». Comptes Rendus Biologies, $\mathrm{n}^{\circ}$ 331, 452-465.

MARGALEF, R. (1974): Ecología. Barcelona. Ed. Omega.

MATHIEU, R., FREEMAN, C. y ARYAL, J. (2007): «Mapping private gardens in urban areas using object oriented techniques and very high-resolution satellite imagery». Landscape and Urban Planning, $\mathrm{n}^{\circ}$ 81, 179-192. 
MCDONNELL, M. y HAHS, A. (2008): «The use of gradient analysis studies in advancing our understanding of the ecology of urbanizing landscapes: current status and future directions». Landscape Ecology, ${ }^{\circ}$ 23, 1143-1155.

MCDONNELL, M.J. y PICKETT, S.T.A. (1990): «Ecosystem structure and function along urban-rural gradients: an unexploited opportunity for ecology». Ecology, n 71, 1232 1237.

MCINTYRE, N.E., KNOWLES-YANEZ, K. y HOPE, D. (2000): «Urban ecology as an interdisciplinary field: differences in the use of «urban» between the social and natural sciences». Urban Ecosystems, n ${ }^{\circ}$ 4, 5-24.

MCKINNEY, M.L. (2002): «Urbanization, biodiversity, and conservation». BioScience, $\mathrm{n}^{\circ}$ 52, 883-890.

MCKINNEY, M.L. (2006): «Urbanization as a major cause of biotic homogenization». Biological Conservation, $\mathrm{n}^{\circ} 127,247-260$.

MCKINNEY, M.L. (2008): «Effects of urbanization on species richness: a review of plants and animals». Urban Ecosystems, $\mathrm{n}^{\circ}$ 11, 161-176.

MCPHERSON, E.G., SIMPSON, J.R. y LIVINGSTON, M., (1989): «Effects of 3 landscape treatments on residential energy and water-use in Tucson, Arizona». Energy and Buildings, $\mathrm{n}^{\mathrm{o}} 13,127-138$.

MENDES, S., COLINO-RABANAL, V.J. y PERIS S. J. (2011): «Bird song variations along an urban gradient: The case of the European blackbird (Turdus merula)». Landscape and Urban Planning, n ${ }^{\circ}$ 99, 51-57.

MILLER, J.R. (2005): «Biodiversity conservation and the extinction of experience». Trends in Ecology and Evolution, $\mathrm{n}^{\mathrm{O}} 20,430-434$.

MILLER, J.R. y HOBBS, R.J. (2002): «Conservation where people live and work». Conservation Biology, $\mathrm{n}^{\circ}$ 16, 330-337.

MUÑOZ, F. (2003): «Lock living: Urban sprawl in Mediterranean cities». Cities, $\mathrm{n}^{\circ} 20$, 381- 385.

MUÑOZ, F. (2007): «La producción residencial de baja densidad en la provincia de Barcelona (1985-2001)» en La ciudad de baja densidad: lógicas, gestión y contención, (F. Monclús, coord.). Barcelona: Diputació de Barcelona, Xarxa de Municipis, 51-84.

MURGUI, E. (2009): «Seasonal patterns of habitat selection of the House Sparrow Passer domesticus in the urban landscape of Valencia (Spain)». Journal of Ornithology, $\mathrm{n}^{\circ} 150$, 85-94.

NACIONES UNIDAS (2012): Convenio sobre la Diversidad Biológica. Doc. UNEP/ CBD/94/1 Rio de Janeiro, Brasil.

OLIVIER, D.F. (1999): «Please, more real farmers in our cities!». Faith \& Earthkeeping, n ${ }^{\circ}$ $16,1-13$.

OWEN, J. (1991): The ecology of a garden: the first fifteen years. Cambridge. Cambridge University Press.

PAOLETTI, E. (2009): «Ozone and urban forests in Italy». Environmental Pollution, $\mathrm{n}^{\circ}$ 157, 1506-1512.

PARLANGE, M. (1998): «The city as ecosystem». BioScience, n 48, 581-585.

PATAKI, D.E., CARREIRO, M.M., CHERRIER, J., GRULKE, N.E., JENNINGS, V., PINCETL, S., POUYAT, R.V., WHITLOW, T.H. y ZIPPERER, W.C. (2011): «Coupling bio- 
geochemical cycles in urban environments: ecosystem services, green solutions, and misconceptions». Frontiers in Ecology and the Environment, $\mathrm{n}^{\circ}$ 9, 27-36.

PAULEIT, S. y DUHME, F. (2000): «Assessing the environmental performance of land cover types for urban planning». Landscape and Urban Planning, $\mathrm{n}^{\circ}$ 52, 1-20.

PICKETT, S.T.A., CADENASSO, M.L., GROVE, J.M., BOONE, G., GROFFMAN, P.M., IRWIN, E., KAUSHAL, S. S., MARSHALL, V., MCGRATH, B.P., NILON, C.H., POUYAT, R.V., SZLAVECZ, K., TROY, A. y WARREN, P. (2011): «Urban ecological systems: Scientific foundations and a decade of progress». Journal of Environmental Management, $\mathrm{n}^{\circ}$ 92, 331-362.

PICKETT, S.T.A., CADENASSO, M.L., GROVE, J.M., NILON, C.H., POUYART, R.V., ZIPPERER, W.C. y COSTANZA, R. (2001): «Urban ecological systems: linking terrestrial ecological, physical and socioeconomic components of metropolitan areas». Annual Review of Ecology and Systematics, $\mathrm{n}^{\circ} 32,127-157$.

PINO, J. y MARULL, J. (2012): «Ecological networks. Are they enough for connectivity conservation? A case study in the Barcelona Metropolitan Region (NE Spain)». Land Use Policy, n 29, 648-690.

PYŠEK, P., J. SADLO, y MANDAK, B. (2002): «Catalogue of alien plants of the Czech Republic». Preslia, $\mathrm{n}^{\circ}$ 74, 97-186.

REICHARD, S.H. y WHITE, P. (2001): «Horticulture as a pathway of invasive plant introductions in the United States». BioScience, $\mathrm{n}^{\circ}$ 51, 103-113.

RIBAS, A., CALBÓ, J., LLAUSÀS, A. y LOPEZ-BUSTINS, J. (2010): «Climate Change at the Local Scale: Trends, Impacts and Adaptations in a Northwestern Mediterranean Region (Costa Brava, NE Iberian Peninsula)». The International Journal of Climate Change; Impacts \& Responses, Vol. 2, 247-264.

RODRÍGUEZ-RODRÍGUEZ, D. (2012): «Integrated networks. A territorial planning proposal for biodiversity conservation in urban, densely populated regions. The case of the Autonomous Region of Madrid, Spain». Journal of Environmental Planning and Management, $\mathrm{n}^{\circ} 55,667-683$.

ROY CHOWDHURY, R. R., LARSON, K., GROVE, M., POLSKY, C., y COOK, E. (2011): «A multi-scalar approach to theorizing socio-ecological dynamics of urban residential landscapes». Cities and the Environment (CATE), Vol. 4, Issue 1.

RUEDA, S. (1995): Ecologia Urbana. Barcelona i la seva regió metropolitana com a referents. Barcelona. Beta Editorial.

SALA, O.E., CHAPIN, F.S. III, ARMESTO, J.J., BERLOW, E., BLOOMFIELD, J., DIRZO, R., HUBER-SANWALD, E., HUENNEKE, L.F., JACKSON, R.B., KINZIG, A., LEEMANS, R., LODGE, D.M., MOONEY, H.A., OESTERHELD, M., POFF, N.L., SYKES, M.T., WALKER, B.H., WALKER, M. y WALL, D.H. (2000): «Global biodiversity scenarios for the year 2100». Science, $\mathrm{n}^{\circ} 287,1770-74$.

SALVADOR, R., BAUTISTA-CAPETILLO, C. Y PLAYÁN E. (2011): «Irrigation perfomance in private urban landscapes: A study case in Zaragoza (Spain)». Landscape and Urban Planning, $\mathrm{n}^{\circ}$ 100, 302-311.

SÁNCHEZ DE LORENZO-CÁCERES, J. M. (coord.). (2000-2010): Flora ornamental española: las plantas cultivadas en la España peninsular e insular. Sevilla. Junta de Andalucía, Consejería de Agricultura y Pesca. 
SANZ-ELORZA, M., DANA E. D. y SOBRINO E., eds. (2004): Atlas de las plantas alóctonas invasoras en España. Madrid, Dirección General para la Biodiversidad, 384 pp.

SAX, D.F. y GAINES, S.D. (2003): «Species diversity: from global decreases to local increases». Trends in Ecology and Evolution, $\mathrm{n}^{\circ}$ 18, 561-566.

SHACKLETON, S.E., CAMPBELL, B., LOTZ-SISITKA, H. y SHACKLETON, C.M. (2008): «Links between the local trade in natural products, livelihoods and poverty alleviation in a semi-arid region of South Africa». World Development, $\mathrm{n}^{\circ} 36,505-526$.

SMITH, R.M., THOMPSON, K., HODGSON, J.G., WARREN, P.H. y GASTON, K.J. (2006): «Urban domestic gardens (IX): Composition and richness of the vascular plant flora, and implications for native biodiversity». Biological Conservation, $\mathrm{n}^{\circ} 129,312-$ 322.

STEWART, I.D. (2011): «A systematic review and scientific critique of methodology in modern urban heat island literatura». International Journal of Climatology, $\mathrm{n}^{\circ}$ 31, 200217.

SULLIVAN, J.J., TIMMINS, S.M. y WILLIAMS, P.A. (2005): «Movement of exotic plants into coastal native forests from gardens in northern New Zealand». New Zealand Journal of Ecology, n 29, 1-10.

TANSLEY, A.G. (1935): «The use and abuse of vegetational concepts and terms». Ecology, $\mathrm{n}^{\mathrm{o}} 16,284-387$.

TERRADAS, J. (2001): Ecologia Urbana. Barcelona. Rubes Editorial.

TROY, A.R., GROVE, J.M., O'NEIL-DUNNE, J.P.M., PICKETT, S.T.A. y CADENASSO, M.L. (2007): «Predicting opportunities for greening and patterns of vegetation on private urban lands». Environmental Management, n 40, 394-412.

ULRICH, R.S. (1981): «Natural versus urban sciences: some psycho-physiological effects». Environment and Behavior, $\mathrm{n}^{\circ} 13,523-556$.

UNITED NATIONS (2013): World Population Prospects: The 2012 Revision, Highlights and Advance Tables. Department of Economic and Social Affairs, Population Division. ESA/P/WP.228

VITOUSEK, P.M., MOONEY, H.A., LUBCHENCO, J. y MELILLO, J.M. (1997): «Human domination of earth's ecosystems». Science, n $277,494-499$.

WALBRIDGE, M.R. (1997): «Urban ecosystems». Urban Ecosystems, nº 1, 1-2.

WHITTAKER, R.H. (1967): «Gradient analysis of vegetation». Biological Reviews of the Cambridge Philosophical Society, $\mathrm{n}^{\circ}$ 49, 207-264.

WILLIAMS, N.S.G., SCHWARTZ, M.W., VESK, P.A., MCCARTHY, M.A., HAHS, A.K., CLEMATIS, S.E., CORLETT, R.T., DUNCAN, R.P., NORTON, B.A., THOMPSON, K. y MCDONNELL, M.J. (2009): «A conceptual framework for predicting the effects of urban environments on flores». Journal of Ecology, n 97, 4-9.

WU, J. (2008): «Making the case for Landscape Ecology: an effective approach to urban sustainability». Landscape Journal, $\mathrm{n}^{\circ}$ 27, 41-50.

WU, J., JENERETTE, G.D. y DAVID, J. (2003): Linking land-use change with ecosystem processes: a hierarchical patch dynamic model. Integrated land use and environmental models. Berlin. Springer, 99-119.

XIAO, Q., y MCPHERSON, E.G. (2002): «Rainfall interception by Santa Monica’s municipal urban forest». Urban Ecosystems, n 6, 291-302. 
